and $y=y_{\mathrm{II}}$ are two such solutions for $\lambda=\lambda_{\mathrm{I}}$ and $\lambda=\lambda_{\mathrm{II}}$ respectively, then one has the relation

$$
\int_{a}^{b} r(x) y_{\mathrm{I}} y_{\mathrm{II}} d x=\left.\frac{y_{\mathrm{II}}^{\prime} y_{\mathrm{I}}-y_{\mathrm{I}}^{\prime} y_{\mathrm{II}}}{\lambda_{\mathrm{I}}-\lambda_{\mathrm{II}}}\right|_{a} ^{b}
$$

(for a derivation see Ref. 1). Considering $y$ as a function of $x$ and $\lambda$, one obtains by the limiting process $\lambda_{\mathrm{II}} \rightarrow \lambda_{\mathrm{I}}, y_{\mathrm{II}} \rightarrow y_{\mathrm{I}}$, that

$$
\int_{a}^{b} r(x) y^{2} d x=\left.\left(\frac{\partial y}{\partial x} \cdot \frac{\partial y}{\partial \lambda}-\frac{\partial^{2} y}{\partial x \partial \lambda} \cdot y\right)\right|_{a} ^{b}
$$

If now the function $y$ is an eigen function and its dependence upon $\lambda$ is explicitly known, this formula yields the normalization constant $\int_{a}^{b} r(x) y^{2} d x$ immediately. Examples are the Jacobi polynomials or the functions of Ref. 2. In either case the functions $y$ are expressed by hypergeometric series, the endpoints of the interval are singular points. The formula may even be useful in problems for which the eigen functions are determined by numerical integration for different values of $\lambda$ and subsequent interpolation.

\title{
REFERENCES
}

1. R. Courant and D. Hilbert, Methoden der mathematischen Physik, Interscience Publishers, New York, Vol. 1, Chap. V, Sec. 3.

2. G. Guderley, Two-dimensional flow patterns with a free stream Mach number close to one, A. F. Technical Report No. 6285.

\section{A NOTE ON THE HODOGRAPH TRANSFORMATION*}

By A. R. MANWELL (University College Swansea)

The hodograph transformation $[1,2]$ for plane compressible flow is too well known to need any discussion here, whilst its singularities have been very fully investigated in $[3,4,5]$. Briefly, it has been found that in cases where plane potential flow is impossible, the continuation of the solution in the hodograph plane may be regular but cannot be mapped into the physical solution. In any given hodograph solution there is no difficulty in deciding if a solution is mappable, for the condition of the vanishing of the Jacobian of the transformation is just that the level lines of the stream function in hodograph space should touch the fixed characteristics in the hodograph space. This criterion of course requires the calculation of all the level lines. Beyond this not much is known, but it has also been shown by various writers, particularly Friedrichs [6], that limit lines cannot appear initially in a certain sense at points inside the solutions. The purpose of this note is to show that the somewhat abstract results of Friedrichs, may be included in the simpler and rather more general statement, that for a wide class of hodograph solutions, if the boundary streamline can be mapped then so can the whole field.

*Received June 15, 1951. 
1. The hodograph equations in characteristic form. The equations of isentropic plane potential flow are

$$
\begin{gathered}
(\rho u)_{x}+(\rho v)_{y}=0 \\
a_{y}-v_{x}=0 \\
\int \frac{d p}{\rho}+\frac{1}{2}\left(u^{2}+v^{2}\right)=\text { const., } \\
\frac{d p}{d \rho}=c^{2}
\end{gathered}
$$

If

and

$$
\begin{gathered}
u=-\psi_{y} / \rho=-\varphi_{x}, \\
v=\psi_{x} / \rho=-\varphi_{y},
\end{gathered}
$$

It is an elementary calculation to verify the equivalence of

$$
\begin{aligned}
& \frac{1}{J}\left\{\varphi_{a}+\frac{(\rho q)_{a}}{\rho^{2} q} \psi_{\theta}\right\}=0, \\
& \frac{1}{J}\left\{\varphi_{\theta}-\frac{q}{\rho} \psi_{a}\right\}=0
\end{aligned}
$$

where

$$
J=\rho^{-2} q^{-1}\left[\psi_{a}^{2}+\frac{\left(1-q^{2} / c^{2}\right)}{q^{2}} \psi_{\theta}^{2}\right]
$$

(c.f. [4] Sec. 103).

Another elementary calculation leads to the characteristic equations equivalent to (1.7), (1.8) viz.

$$
\begin{gathered}
U_{\alpha}+\frac{1}{2} B(V-U)=0 \\
V_{\beta}+\frac{1}{2} B(U-V)=0
\end{gathered}
$$

where

$$
\begin{aligned}
& 2 d \alpha=d r / r-d \theta=q^{-1}\left(q^{2} / c^{2}-1\right)^{1 / 2} d q-d \theta \\
& 2 d \beta=d r / r+d \theta
\end{aligned}
$$

and

$$
U=\psi_{\beta} / h \quad V=\psi_{\alpha} / h,
$$

where $h^{2}\left(q^{2} / c^{2}-1\right)=\rho^{2}$. 
Also.

$$
B=-r h^{\prime} / h=\frac{1}{2}(\gamma+1) \frac{q^{4} / c^{4}}{\left[q^{2} / c^{2}-1\right]^{3 / 2}}
$$

and $B$ is positive if it is agreed that the sign of the radical is to be chosen so that $r$ increases with $q$. In this case both $\alpha$ and $\beta$ increase with $q$. Now in the supersonic region the sign of $J=U V / q^{3}$ is that of $U V$, and for a continuous streamfunction defined in a simply connected region which is partly subsonic, the problem of showing that the solution can be mapped into physical space reduces to showing that $U V$ is positive in the supersonic region. It will henceforth be further assumed that $U$ and $V$ are also continuous and piecewise analytic. Then if $J$ changes sign it must vanish and, according to the theory of hyperbolic differential equations, discontinuities in the derivatives of $U$ and $V$ can occur only across characteristics, so that there will be Taylor expansions for $U, V$ in each quadrant of the $\alpha, \beta$ plane near the zeros of $J$. It will however be sufficient for the present purpose to consider the behaviour on the characteristics themselves.

2. Expansions near a zero of $J$. Let $\alpha, \beta$ be, for the sake of convenience, measured from the point where $J=0$. The cases that only one or both of $U, V$ vanish must be considered separately.

Suppose for example that $U \neq 0$ whilst $V=0$ and along $\alpha=$ const $=0 V=$ $V(\beta)$. Equation (1.11) shows that

$$
V(\beta) \cong-\frac{1}{2} B_{0} U_{0} \beta,
$$

where the suffix 0 refers to the zero. Therefore

$$
U(\beta) V(\beta) \cong-\frac{1}{2} B_{0} U_{0}^{2} \beta,
$$

and since $B>0$ everywhere the sign of $J$, assumed positive in this paper for the subsonic region, is fixed with respect to increasing $q$.

Similarly, if $U=0$ but $V \neq 0$,

$$
U(\alpha) V(\alpha) \cong-\frac{1}{2} B_{0} V_{0}^{2} \alpha .
$$

If on the other hand both $U$ and $V$ vanish and so $V(\alpha) \cong V_{n} \alpha^{n}$ with $n>0$, (1.10) gives $U(\alpha) \cong-\frac{1}{2} V_{n} B_{0} \alpha^{n+1}$ so that

$$
U(\alpha) V(\alpha) \cong-\frac{1}{2} V_{n}^{2} B_{0} \alpha^{2 n+1}
$$

whilst similarly from (1.11)

$$
U(\beta) V(\beta) \cong-\frac{1}{2} V_{m}^{2} B_{0} \beta^{2 m+1}
$$

It is perhaps necessary to point out that in the full Taylor expansions of $U, V$ there may well be terms of lower order than $m$ or $n$ and that this is associated with cusped limit lines enclosing the axes $\alpha=0$ and $\beta=0$ near the zero. Equations (2.2), (2.3), (2.4) show that $J$ is strictly decreasing with increasing $q$ along at least one of the characteristic directions through a point where $J=0$. Its behaviour along general directions cannot however be predicted.

At a sonic point the characteristic equations (1.10), (1.11) cannot be used directly but it is still desirable to retain this form as far as possible. To do this the limit of the previous equations will be considered for points near the sonic line.

Let $q=c_{*}(1+t)$ where $c_{*}$ is the critical speed and $t$ small and positive. 
Then

$$
\begin{aligned}
c^{2} & \simeq c_{*}^{2}[1-(\gamma-1) t] \\
h & \simeq \rho_{*} /\left[(1+\gamma) t^{3}\right]^{1 / 2}, \\
r & =r_{0}\left[1+O\left(t^{3 / 2}\right)\right], \\
B & \simeq \frac{1}{2}\left[(1+\gamma) t^{3}\right]^{-1 / 2},
\end{aligned}
$$

while

$$
\begin{aligned}
& U \simeq \rho_{*}^{-1}\left\{\psi_{t}+[(1+\gamma) t]^{1 / 2} \psi_{\theta}\right\} \\
& V \simeq \rho_{*}^{-1}\left\{\psi_{t}-[(1+\gamma) t]^{1 / 2} \psi_{\theta}\right\}
\end{aligned}
$$

and

$$
d \alpha \simeq d \beta \simeq[(1+\gamma) t]^{1 / 2} d t .
$$

If $J$ vanishes $\left(\psi_{t}\right)=0$ and near the zero on the sonic line the expansions along either characteristic are of the form $U \cong P t^{m}$ and $V \cong Q t^{n}$ where $m$ and $n$ are not less than $\frac{1}{2}$.

1.10 (or 1.11) after multiplication by $t^{\frac{1}{2}}$ and integration from 0 to $t$ gives either $m=n$ and $Q=(4 n-1) P$ or $m>n$ and $(4 n-1)=0$; the second possibility must be rejected. Hence

$$
J \simeq-(4 n-1) \rho^{2} r_{0}^{2} t^{2 n} / c^{2}
$$

and there is a similar but in general distinct expansion on the other characteristic.

From 2.3, 2.4, 2.8 it appears that near a zero of $J$ it changes sign positive to negative along at least one characteristic in the direction of increasing $q$. The absolute sign in the subsonic region must of course be taken as positive.

This contains the basic result found in [1] that near a zero of $J$ it must change sign, and the conditions on the solution here are slightly less restrictive.

Let the flow, or one sheet of it, be bounded by a smooth non-characteristic streamline $C$ on which it will be further assumed that $\partial \psi / \partial n$ is of one sign in the supersonic region. $C$ will have one-one correspondence with $\alpha, \beta$ and $J$ will be positive on $C$. Let $\alpha_{m}$ be the largest value of $\alpha$ for which $J$ vanishes in the solution inside $C$ and choose on $\alpha=\alpha_{m}$ the particular zero $K$ with maximum $\beta$. Now $K$ cannot lie on $C$ and from the form of the region in the $\alpha, \beta$ plane it is possible to join it to $C$ by two characteristic arcs each in the direction of increasing $q$. On these $\operatorname{arcs} J>0$ by the construction whilst it has already been proved that near $K J$ is essentially negative. It is therefore impossible for $J$ to vanish inside the region.

Figure 1(a) shows the impossible case of isolated zeros disproved by Friedrichs, Fig. 1(b) the more general situation excluded by this theorem.

There are two simple corollaries to this conclusion:-

(i) For solutions of the above type the streamfunction cannot have a maximum or minimum at interior points of the region. It is only necessary to show this for the supersonic part and there if $\psi_{a}=0$ and $\psi_{\theta}=0$ then $J=0$ also which is impossible.

(ii) The uniqueness theorem for Cauchy data on part of $C$.

Let $\left(U_{1}, V_{1}\right)$ and $\left(U_{2}, V_{2}\right)$ be two solutions of the above type and if at some point 


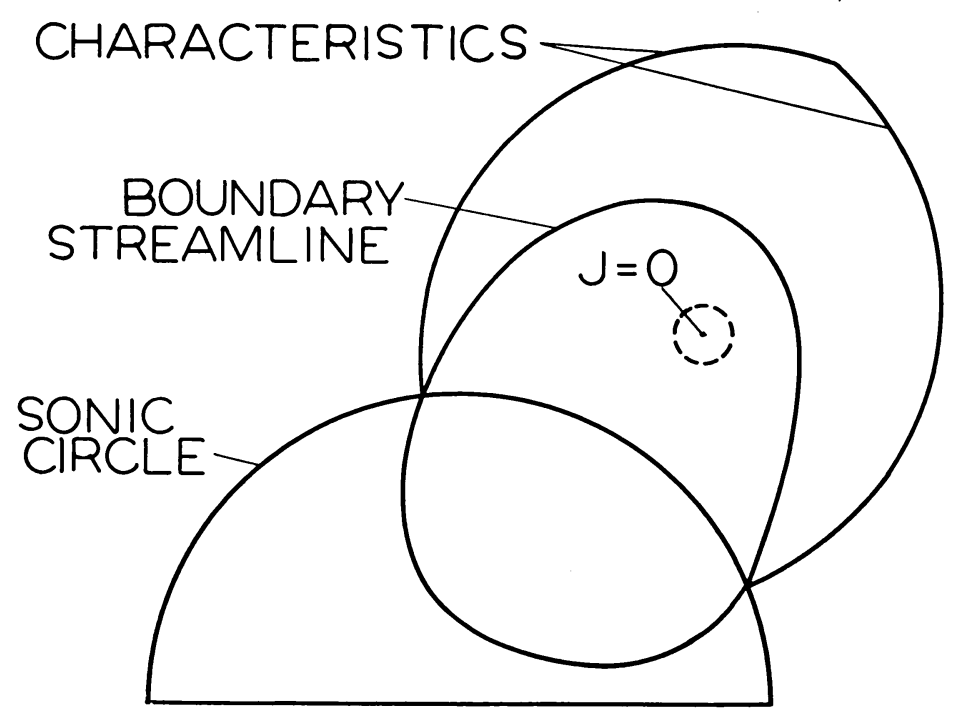

FIG. 1(a). Hodograph plane: $J$ cannot be positive on the entire broken line in the vicinity of $J=0$.

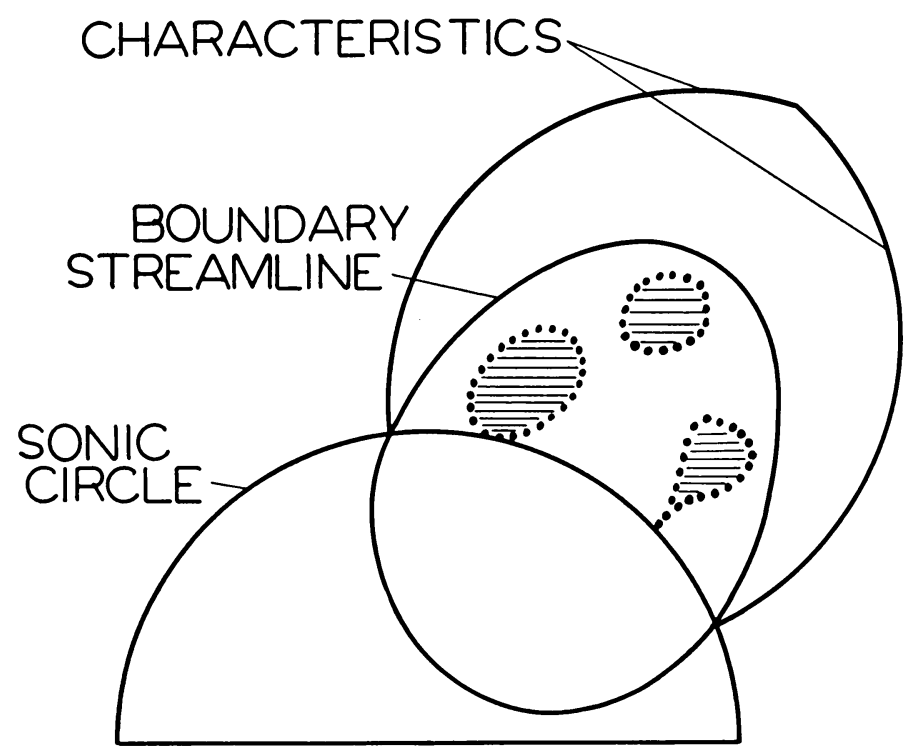

FIG. 1(b). Hodograph plane: Limit lines, shown dotted, enclosing shaded regions of $J<0$.

inside the region $U_{1} \neq U_{2}$ (say) i.e. $U_{1}=\lambda U_{2}$ with $\lambda \neq 1$ the solution

$$
(\bar{U}, \bar{V})=\left[\frac{U_{1}-\lambda U_{2}}{1-\lambda}, \frac{V_{1}-\lambda V_{2}}{1-\lambda}\right]
$$

has the same Cauchy data on the arc $C$ as the original solutions. However, by construction, $J$ for the new solution vanishes at one point inside the flow whilst the above theorem applies giving a contradiction. The region in which uniqueness has been proved 
may be a triangle lying entirely in the supersonic region or it may be a quadrilateral extending from the arc of $C$ to the sonic line. (Fig. 2).

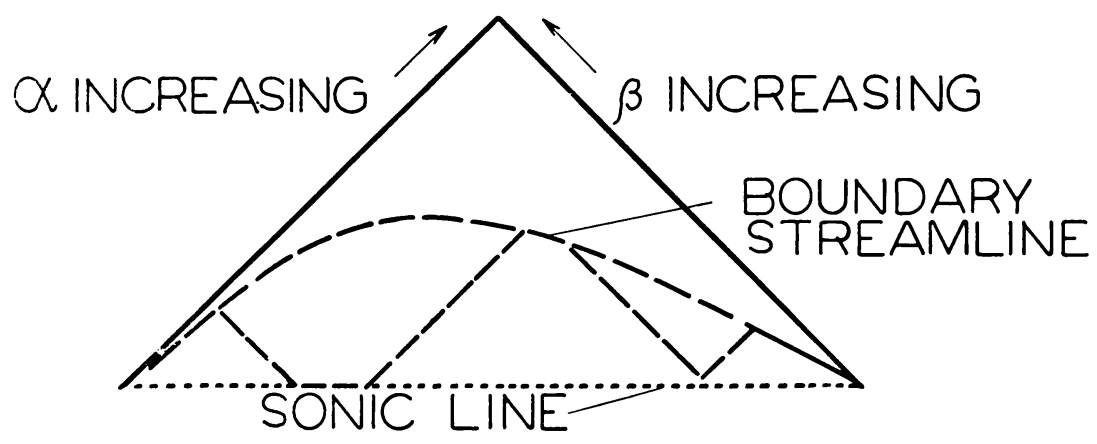

FIG. 2. Characteristic plane: Broken lines indicate typical boundaries for uniqueness with Cauchy data on the corresponding streamline arc.

3. Solutions in the physical plane. If a hodograph solution has been found which satisfies the mapping condition then an infinity of neighbouring solutions can be constructed which are also mappable. For, let $\psi_{1}(q, \theta)$ be another solution having the same singularities and branch points in the subsonic region and, if $\epsilon \psi_{1}$ is added to the original solution, in general the boundary streamline and the values of the Jacobian on it will be altered, but, by quantities which by choosing $\epsilon$ may be made as small as is desired. However, it was pointed out in [7] that infinitesimal changes of the physical boundary demand in general finite changes of the hodograph. This, which was shown in detail for subsonic flow is generally true. It is a consequence of there being no unit of length in the equations of motion of an inviscid fluid so that flow patterns do not depend on the scale of figure. Any small disturbance of the physical boundary is equivalent locally to the insertion of one half of a symmetrical aerofoil into the wall of a wind-tunnel. Since it is known that not all symmetrical aerofoils permit continuous flow it follows that general small displacements of the boundary will lead to shock waves. For subsonic flow however the variations must have large changes of slope and the disturbances will be merely local.

In the case of local supersonic flow the situation is rather different. That some small variations of the physical boundary will lead to an end of continuous flow is a consequence of the existence of any symmetrical profile e.g. a very flat double wedge over which continuous flow has been proved impossible. Moreover the disturbances will be propagated along nearly characteristic paths in the physical plane to finite distances (Ref. 5, pp. 54, 131). Thus, whilst in the strict mathematical sense all compressible flows are "unstable" in that near boundaries cannot have shock free flow, for transonic flow the "instability" has a physical meaning. C.f. $[8,9]$.

By means of the theorem of this note the situation for flows with simple hodographs can be examined in more detail. (In physical flows there may also be both simple waves and flows needing several sheets in the hodograph). In any neighbouring solution corresponding to a small bulge in the physical plane only a few streamlines are affected. In the hodograph, which is supposed simple, these must initially pass into a new part of the plane. Also since the disturbance of the whole flow is small they leave through a small 
gap in the hodograph boundary. On the other hand for finite changes of slope over the bulge they then must spread over a finite region of the hodograph plane. With these requirements it is, see Fig. 3, not possible to draw a smooth mappable hodograph boundary for the disturbed flow.

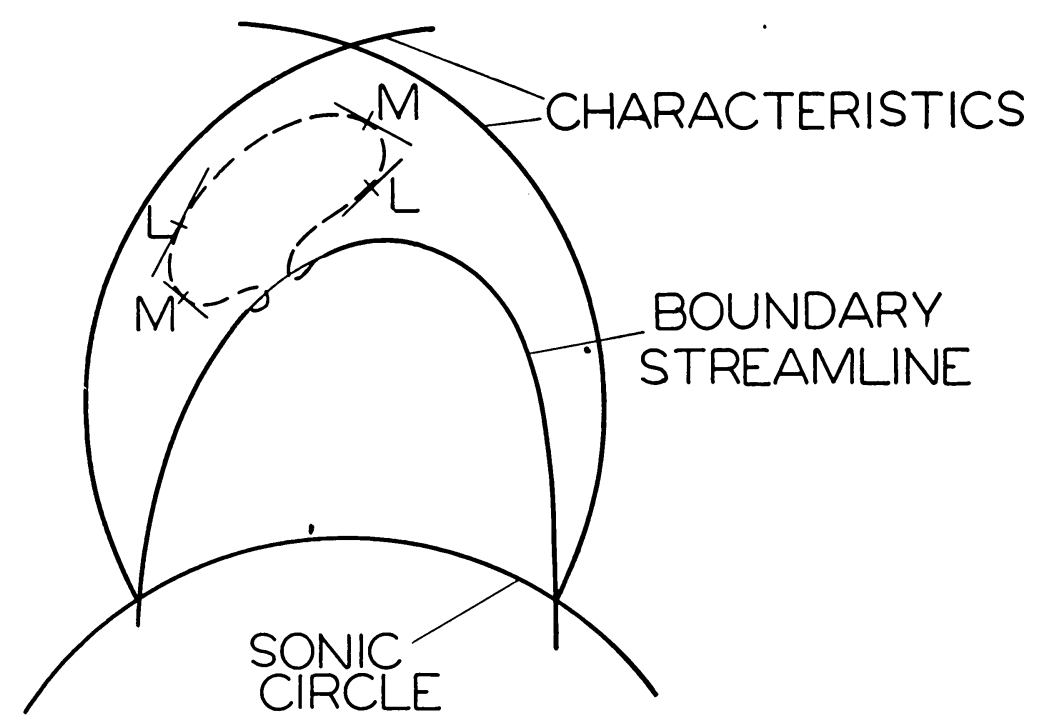

FIG. 3. Hodograph plane: Limit lines appear at $L, M$ on a typical perturbed boundary streamline, shown by broken line.

Leaving aside the question of "stability" which, in situations in which shocks occur in a continuous manner, may not be of vital importance, one may enquire what theoretical limitations the theorem of this paper puts on simple hodograph solutions. The answer to this question depends on the shape of the nose of the aerofoil. Usually this is normal to the stream and the boundary velocity up to the mid-section is turned through $90^{\circ}$. Comparison with the limiting characteristic shows that $q / c^{*}$ is limited to 2.24. If the nose of the aerofoil is cusped inwards the boundary velocity can turn through $180^{\circ}$, and the velocity increase found by tracing a characteristic through an angle $(\sqrt{6}-1) \pi / 2$ radians $\left[130^{\circ}\right]$ gives $q / c^{*}=\sqrt{6}$, the value for cavitation with $\gamma=1.4$. Therefore the theorem places no new limit on the local Mach numbers possible in transonic flow and it seems quite likely that mathematical solutions with cavitation do in fact exist.

Conclusion. The author first proved the theorem of this paper for the case that only one factor of $J$ vanishes at each point on the limit line. This case, which is the usual one in applications, can be proved by showing that closed limit lines inside the flow are not possible whilst by hypothesis they cannot end on the boundary $C$. The proof given here was however arrived at after a study of reference [1] which also suggested the more general result given here. The discussion of the physical "stability" is largely independent of this theorem depending on the principle of the similitude of compressible flows at the same Mach numbers but different Reynold's numbers viscosity being neglected in the mathematical theory. 
The author also gratefully acknowledges the help of Professor W. R. Sears and Dr. Kuo in studying an earlier draft of this paper and in particular their advice regarding the section on the physical solutions.

\section{REFERENCES}

1. P. Molenbroek, Über einige Bewegungen eines Gases bei Annahme eines Geschwindigkeitspotentials, Archiv. Math. Phys. 9, 157-195 (1890).

2. S. A. Chaplygin, On gas jets, Ann. Imp. Univ. Mosc. (Phys-Math.) 21, 1-121 (1904).

3. H. S. Tsien, The limiting line in mixed subsonic and supersonic flow of compressible fluids, N.A.C.A. Tech. Note 961 (1944).

4. R. Courant and K. O. Friedrichs, Supersonic flow and shock waves, Interscience Publishers Inc., New York, 1948, p. 62.

5. J. Craggs, The breakdown of the hodograph transformation, Proc. Camb. Phil. Soc. 44, 360-379 (1948).

6. K. O. Friedrichs and D. Flanders, On the non-occurrence of a limiting line in transonic flow, Com. App. Math. (1) 3, 287-301 (1948).

7. A. R. Manwell, A method of variation for flow problems, Quart. Journ. Math. (Oxford) 20, 166-189 (1949).

8. F. I. Frankl, On the formation of shock waves in subsonic flows with local supersonic velocities, (Transl.), N.A.C.A. Tech. Mem. 1251.

9. G. Guderley, On the transition from a transonic potential flow to a flow with shocks, Air. Mat. Comm., T-2, F-TR-2160, ND.

\section{NOTE ON A SUFFICIENT CONDITION FOR THE STABILITY OF GENERAL, PLANE PARALLEL FLOWS*}

By MARTIN LESSEN (The Pennsylvania State College)

In reference 1, Synge derived sufficient conditions for the stability of plane Couette motion and plane Poiseuille motion. In the present note, it is demonstrated that similar conditions exist for any parallel flow with either finite or infinite boundary conditions or both. Although it can be shown that parallel two-dimensional motion must be of either Poiseuille or Couette type, it has been assumed, for purposes of stability considerations, that boundary-layer flows are substantially parallel in nature and Synge's treatment has therefore been extended to cover these flows.

The equation describing the stability of parallel flows for small disturbances is the Orr-Sommerfeld equation (reference 2 ):

$$
(w-c)\left(\varphi^{\prime \prime}-\alpha^{2} \varphi\right)-w^{\prime \prime} \varphi=-\frac{i}{\alpha R}\left(\varphi^{\mathrm{IV}}-2 \alpha^{2} \varphi^{\prime \prime}+\alpha^{4} \varphi\right),
$$

where $x$ is the coordinate in direction of the flow, $y$ the coordinate perpendicular to this direction, $t$ the time, $w=w(y)$ the velocity of steady flow, $\varphi(y) e^{i \alpha(x-c t)}$ the disturbance stream function; $c$ the disturbance phase velocity, $\alpha$ the disturbance wave number, $R$ the Reynolds number of steady flow.

${ }^{*}$ Received July $2,1951$. 